\title{
Cytotoxicity and apoptosis induced by nanobacteria in human breast cancer cells
}

This article was published in the following Dove Press journal:

International Journal of Nanomedicine

30 December 2013

Number of times this article has been viewed

\section{Ming-jun Zhang' \\ Sheng-nan Liu' \\ $\mathrm{Ge} X \mathrm{u}^{2}$ \\ Ya-nan Guo' \\ Jian-nan $\mathrm{Fu}^{3}$ \\ De-chun Zhang'}

'Molecular Medicine and Tumor Research Center, Chongqing Medical University, ${ }^{2}$ Electron Microscopy Group, Department of Life Science, Chongqing Medical University, Chongqing, People's Republic of China; ${ }^{3}$ First People's Hospital of Jiulongpo District, Chongqing, People's Republic of China
Correspondence: De-chun Zhang, Molecular Medicine and Tumor Research Center, Chongqing Medical University, I Medical College Road, Yuzhong District, Chongqing 4000I6, People's Republic of China

Tel +8602368486133

Fax +8602368486133

Email zhangdechun46@I63.com
Background: The existing evidence that nanobacteria (NB) are closely associated with human disease is overwhelming. However, their potential toxicity against cancer cells has not yet been reported. The objective of this study was to investigate the cytotoxic effects of NB and nanohydroxyapatites (nHAPs) against human breast cancer cells and to elucidate the mechanisms of action underlying their cytotoxicity.

Methodology/principal findings: NB were isolated from calcified placental tissue, and nHAPs were artificially synthesized. The viability of the MDA-MB-231 human breast cancer cell line was tested by using the Kit- 8 cell counting kit assay. Apoptosis was examined by transmission electron microscopy and flow cytometry. The endocytosis of NB and nHAPs by MDA-MB231 cells was initially confirmed by microscopy. Although both NB and nHAPs significantly decreased MDA-MB-231 cell viability and increased the population of apoptotic cells, NB were more potent than nHAPs. After 72 hours, NB also caused ultrastructural changes typical of apoptosis, such as chromatin condensation, nuclear fragmentation, nuclear dissolution, mitochondrial swelling, and the formation of apoptotic bodies.

Conclusion/significance: In MDA-MB-231 human breast cancer cells, NB and nHAPs exerted cytotoxic effects that were associated with the induction of apoptosis. The effects exerted by NB were more potent than those induced by nHAPs. NB cytotoxicity probably emerged from toxic metabolites or protein components, rather than merely the hydroxyapatite shells. NB divided during culturing, and similar to cells undergoing binary fission, many NB particles were observed in culture by transmission electron microscopy, suggesting they are live microorganisms.

Keywords: nanobacteria, nanohydroxyapatites, human breast cancer MDA-MB-231 cells, cytotoxicity, apoptosis

\section{Introduction}

Nanobacteria (NB) is a term used to refer to a proposed class of live organisms much smaller in size than what is generally accepted to be the lowest limit for life. ${ }^{1}$ It is still controversial whether NB are truly microorganisms, but they have a unique morphology. These bacteria are 80 to $500 \mathrm{~nm}$ in diameter; typically present coccoid, coccobacillar, or bacillar shapes; have a mineralized, "igloo-like" structure;, and are covered in hydroxyapatite shells. ${ }^{4}$ Moreover, they are self-propagating under conventional cell culturing conditions $\left(37^{\circ} \mathrm{C} ; \mathrm{pH}, 7.4 ; 5 \% \mathrm{CO}_{2^{-}} 95 \%\right.$ air $){ }^{5}$ For the last 2 decades, significant controversies have existed regarding the exact nature of $\mathrm{NB}$, particularly with respect to whether they are self-replicating life-forms or mineralprotein complexes without any relation to bacteria. The theory that NB might represent live microorganisms is based on an unusual set of microbiological characteristics, 
including their slow growth in liquid media, the formation of biomembranes, ${ }^{6}$ their staining with DNA-specific dyes, ${ }^{7}$ and their susceptibility to antibiotics. ${ }^{8}$ However, all these pieces of evidence are indirect, and no accurately sequenced genomes have existed, to date, to establish NB as live organisms. ${ }^{9}$ More important, in certain studies, a number of serum proteins (fetuin A, albumin, lipoprotein, and others) were isolated from NB. ${ }^{10}$ The published nanobacterial $16 \mathrm{~S}$ rRNA sequence was also attributed to occasional environmental Brucella or Bartonella contamination. ${ }^{11}$ These data support the conclusion that NB are actually inorganic materials or, probably, calcium-protein complexes. ${ }^{12}$

In our previous studies, NB were successfully isolated from human placental tissues, and their self-replicating capacity was confirmed by a growth curve. Moreover, we both identified their 16S rRNA (GenBank JF823648), which shares $80 \%$ sequence homology with sequences reported by Kajander's team, ${ }^{1}$ and observed images of their division by transmission electron microscopy (TEM). These pieces of evidence convinced us that NB are microorganisms, rather than inorganic complexes. NB have been proposed to be important in the pathogenesis of various human diseases, especially in association with a variety of pathological calcifications; notably, cholecystolithiasis; bladder, renal, and ureteral lithiasis; placental calcification; psammoma bodies of ovarian cancer; and arterial sclerosis. ${ }^{13-18}$ However, the role of NB in cancer cell growth has not been reported so far. In the current study, we investigated the cytotoxic effects of NB and nanohydroxyapatites (nHAPs) against human MDA-MB231 breast cancer cells and elucidated the mechanisms of action underlying this cytotoxicity.

The protocol was approved by the Ethics Committee of the Chongqing Medical University, Chongqing, People's Republic of China (permit number 2011-2012003).
Written informed consent was obtained from all the study participants. All procedures followed the tenets of the Declaration of Helsinki.

\section{Materials and methods Cell culture}

MDA-MB-231 cells, purchased from the American Type Culture Collection (Manassas, VA, USA; ATCC ${ }^{\circledR}$ HTB26TM), were stored in liquid nitrogen. The frozen vials were thawed by gentle agitation in a $37^{\circ} \mathrm{C}$ water bath immediately after being taken out from the liquid nitrogen tank. Cells were grown in RPMI 1640 medium (Hyclone) containing penicillin (110 IU/mL), streptomycin $(100 \mu \mathrm{g} / \mathrm{mL})$, and $2 \mathrm{mM}$ glutamine and supplemented with $10 \%$ fetal bovine serum (Life technologies Corporation, Grand Island, NY, USA). The cells were routinely cultured in $50 \mathrm{~cm}^{2}$ plastic culture flasks at $37^{\circ} \mathrm{C}$ in a humidified atmosphere with $5 \% \mathrm{CO}_{2^{-}} 95 \%$ air. The medium was refreshed every 24 hours, and cells were trypsinized $(0.1 \%$ trypsin) on reaching $80 \%$ confluency (Figure $1 \mathrm{~A})$.

\section{NB cultures}

NB were isolated from calcified placental tissue (GenBank JF 823648 $)^{19}$ and preserved at $4^{\circ} \mathrm{C}$ in RPMI with $30 \%$ glycerol and $10 \%$ FBS. After centrifugation at $14,000 \times g$ for 40 minutes, the supernatant was removed and pellets were resuspended in $5 \mathrm{~mL}$ RPMI with 10\% FBS. NB were grown for 4 weeks at $37^{\circ} \mathrm{C}$ in a humidified incubator with $5 \% \mathrm{CO}_{2}$, and the media was refreshed every 3 days (Figure 1B). NB growth was monitored with a transmission microscope and with a turbidity meter (LP2000-11; Shanghai Precise Instrument Co, Shanghai, People's Republic of China), and the final NB concentrations were separately adjusted to $0.5,1$, and 2 Meclary turbidity (MCF) just before use. The diameter of NB varied from 80 to $300 \mathrm{~nm}$ under scanning electron microscopy (Figure 2A).
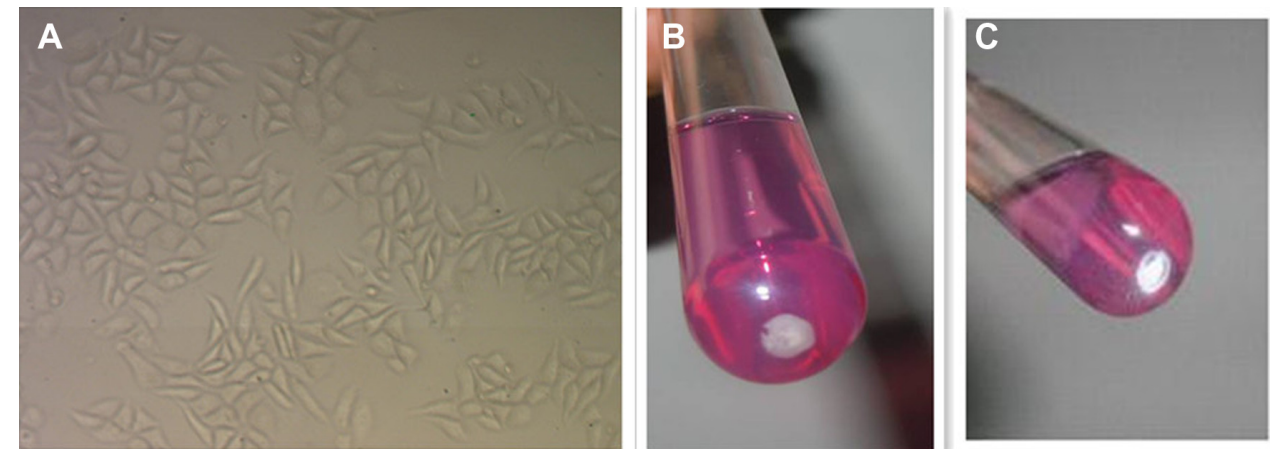

Figure I (A) Morphology of MDA-MB-23I cells observed by using a phase contrast microscope (I00x). (B) Anabiotic nanobacteria (nanobacteria; GenBank JF823648). (C) Nanohydroxyapatites (handled the same way as nanobacteria). 


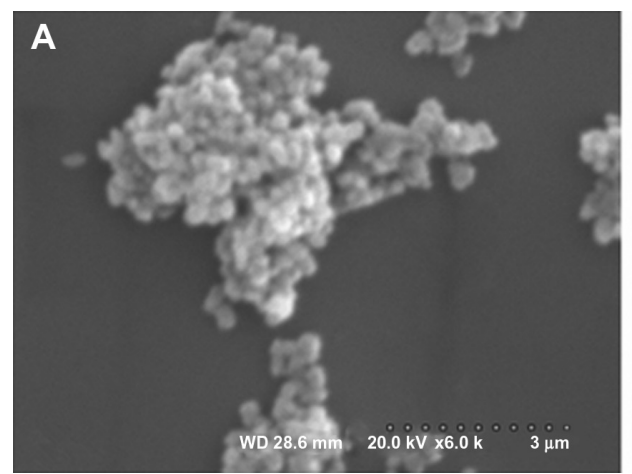

Figure 2 Scanning electron microscopy characterizations of nanoparticles. Notes: (A) Nanobacteria; (B) nanohydroxyapatites.

\section{Preparation of $\mathrm{nHAPs}$}

nHAP solutions (Shangai Baoman Technical Co, Shanghai, People's Republic of China) were prepared by mixing $400 \mu \mathrm{g}$ nHAPs with $2 \mathrm{~mL}$ phosphate buffered saline (PBS; $200 \mu \mathrm{g} / \mathrm{mL}$ ) and ultrasonicating overnight. The solutions were then adjusted with culture media to $100 \mu \mathrm{g} / \mathrm{mL}$ and $50 \mu \mathrm{g} / \mathrm{mL}$ and filtered through $0.22 \mu \mathrm{m}$ filters (Figure 1C). The particle size of nHAPs was determined by scanning electron microscopy and ranged from 50 to $200 \mathrm{~nm}$ (Figure 2B).

\section{Cell viability and proliferation assays}

The effects of nanoparticles on cell viability and proliferation were evaluated using a Kit-8 cell counting kit (CCK-8) (Sigma-Aldrich, St Louis, MO, USA) according to the manufacturer's protocol. MDA-MB-231 cells were seeded on 96-well plates at a density of $2 \times 10^{4}$ cells/well per $100 \mu \mathrm{L}$. The next day, NB (2 MCF) or nHAP (2 MCF) suspensions were added to the culture ( $10 \mu \mathrm{L} /$ well). Untreated and blank groups were set as controls. After 72-hour exposure, the CCK-8 solution was added to the wells at a 500-nM final concentration, and the plates were incubated for an additional 4 hours in a $\mathrm{CO}_{2}$ incubator at $37^{\circ} \mathrm{C}$. The absorbance was measured at $650 \mathrm{nM}$, using a microplate spectrophotometer ( $\mu$ Quant; BioTek Instruments, Inc., Winooski, VT, USA), and adjusted for each corresponding blank (NB plus medium with CCK-8 solution, nHAPs plus medium with CCK-8 solution, and media with CCK-8 solution).

\section{Flow cytometric analysis of apoptosis}

For apoptosis assays by flow cytometry, $1 \times 10^{5}$ MDA-MB231 cells per well were seeded in 6-well plates overnight and then treated for 72 hours with NB or nHAPs. After treatment, cells were trypsinized, washed twice with PBS, and then incubated in the dark for 15 minutes with fluorescein isothiocyanate (FITC)-annexin V and propidium iodide (PI)

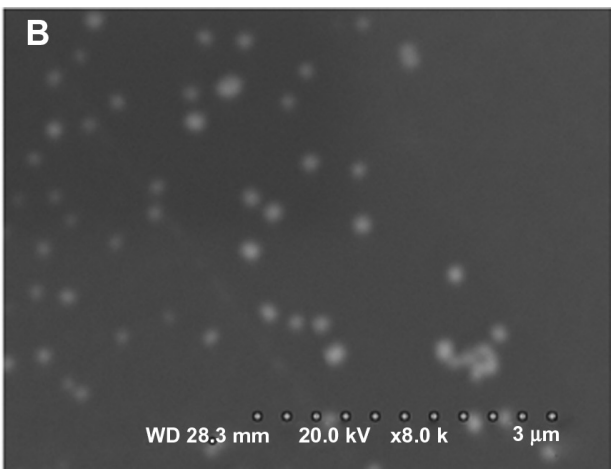

in binding buffer. Stained cells were immediately subjected to fluorescence-activated cell sorting analysis, using a flow cytometer (FCM; compuCyte, Cambridge, MA, USA). The percentage of apoptotic and necrotic cells was calculated from the total cell numbers obtained by the FCM Cell Quest, version 2.2 software (FCM). Double-negative cells are indicative of live cell populations, annexin V-positive cells indicate early apoptotic cells, PI-positive cells indicate necrotic cells, and double-positive cells indicate late apoptotic or necrotic cells.

\section{Evaluation of cell morphology}

The morphological changes of subconfluent MDA-MB231 cells in 96-well plates were evaluated using phase contrast microscopy after 72 hours of treatment with NB and nHAPs.

\section{Electron microscopy}

Subconfluent MDA-MB-231 cells in 6-well plates were exposed to NB or nHAPs for 72 hours, washed with PBS, gently trypsinized, and collected by centrifugation. Cell pellets were washed 3 times in PBS, fixed for 1 hour with 2.5\% glutaraldehyde, and postfixed for 1 hour with $1 \%$ osmic acid. After dehydration in a graded series of cold ethanol, cells were subsequently embedded in epoxy resin. Ultrafine sections were performed, using an ultramicrotome, before staining with uranyl acetate and counterstaining with lead citrate. Sections were then examined with a transmission electron microscope (Hitachi-600; Hitachi Ltd, Tokyo, Japan).

\section{Statistical analysis}

When determining the viability of cells, results were presented as mean \pm standard deviation. Data were analyzed using the SPSS statistical package (version 17; IBM Corporation, Armonk, NY, USA) and PRISM 5 (GraphPad Software, Inc., La Jolla, CA, USA). The $t$-test was used to analyze statistical significance between conditions, and one-way and 

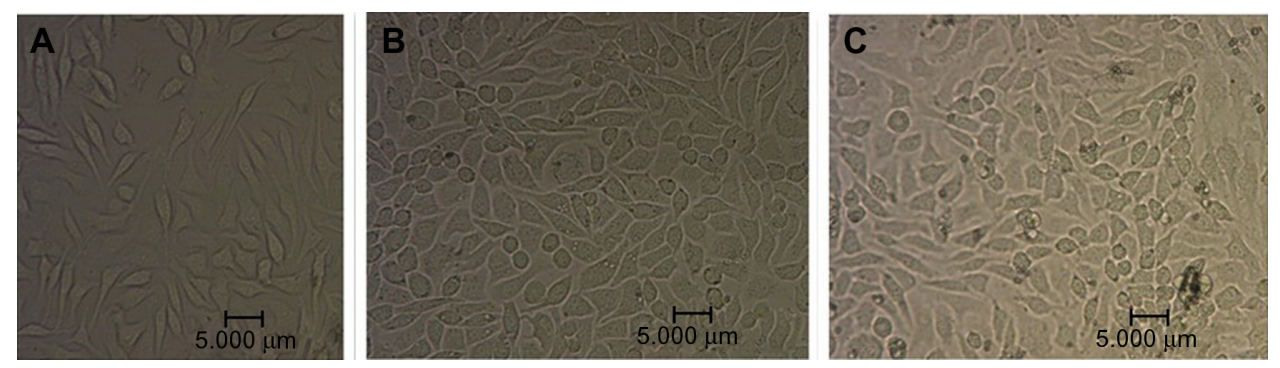

Figure 3 Morphology of breast cancer cells after nanoparticle exposure. MDA-MB-23I morphology was observed using a phase contrast microscope $(\times 400)$. Notes: (A) Control; (B) nanohydroxyapatites; (C) nanobacteria.

two-way analyses of variance were used to analyze statistical results among several groups. We considered $P<0.05$ to be statistically significant.

\section{Results}

\section{Interaction of nanoparticles with MDA-MB-23 I cells}

The effects of NB and nHAPs on MDA-MB-231 cellular morphology were observed under an optical microscope. As shown in Figure 3, MDA-MB-231 cells underwent morphological changes after a 72-hour exposure to nanoparticles. Both NB and nHAPs were endocytosed by the cells. The most conspicuous changes observed in NB- or nHAP-treated cells included an uneven cellular size and cytoplasmic vacuoles. The morphological changes in the NB group were more remarkable than those in the nHAP group. We counted 10 visual fields. The percentages of the cells with large macroscopic cytoplasmic vacuoles and with uneven size were $21.2 \%$ (318/1,509 cells) in the NB group and $5.8 \%(108 / 1,862$ cells) in the nHAP group. There were significant differences between the NB and nHAP groups.

\section{Effects of nanoparticles on MDA-MB-23 I cell proliferation}

We used the CCK-8 assay to monitor cellular proliferation. The data showed that nanoparticles impaired MDA-MB-231 breast cancer proliferation. After 72 hours of treatment, a significant decrease in the number of viable cells (absorbance values) was observed for both the NB and the nHAP groups compared with the control group $(P<0.001)$. NB showed a more prominent reduction in the proliferation of MDA-MB-231 cells than nHAPs (Figure 4), and a significant difference inhibition rate was seen between the two treatment groups $(P<0.001)$.

\section{Induction of apoptosis}

To investigate whether the nanoparticle-induced toxicity in MDA-MB-231 cells is mediated by apoptosis, we treated cells for 72 hours and performed annexin V/PI assay by flow cytometry. As shown in Figure 5, the cell population in the lower right quadrant, which corresponds to the percentage of early apoptotic cells (annexin V-positive and PI-negative), increased to $2.35 \% \pm 0.03 \%$ and $4.14 \% \pm 0.12 \%$ in the presence of nHAPs and NB, respectively, compared with $0.22 \% \pm 0.06 \%$ for control. The percentage of necrotic and late apoptotic cells (both annexin V- and PI-positive cells in the upper right quadrant) were $1.99 \% \pm 0.6 \%$ for nHAPs, $7.45 \% \pm 1.8 \%$ for $\mathrm{NB}$, and $2.2 \% \pm 0.7 \%$ for the control group. The data indicated that NB significantly induced early apoptosis $(P<0.01)$ as well as late apoptosis and necrosis $(P<0.01)$ when compared with the control, whereas nHAPs induced only early apoptosis $(P<0.01)$, not late apoptosis or necrosis $(P>0.05$; Figure 5$)$.

\section{Effects of nanoparticles on cell ultrastructure}

Differences in cellular ultrastructure were observed between MDA-MB-231 cells treated with nanoparticles and control cells. TEM confirmed that nanoparticles were internalized

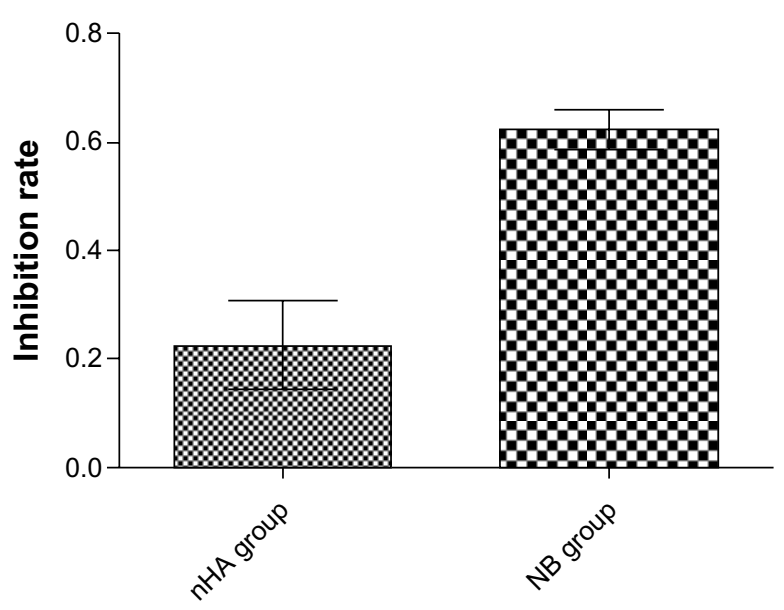

Figure 4 Cytotoxicity of nanoparticles. The effects of nanobacteria (NB) and nanohydroxyapatites $(\mathrm{nHA})$ on cellular proliferation, as determined by the Kit-8 cell counting kit assay. 

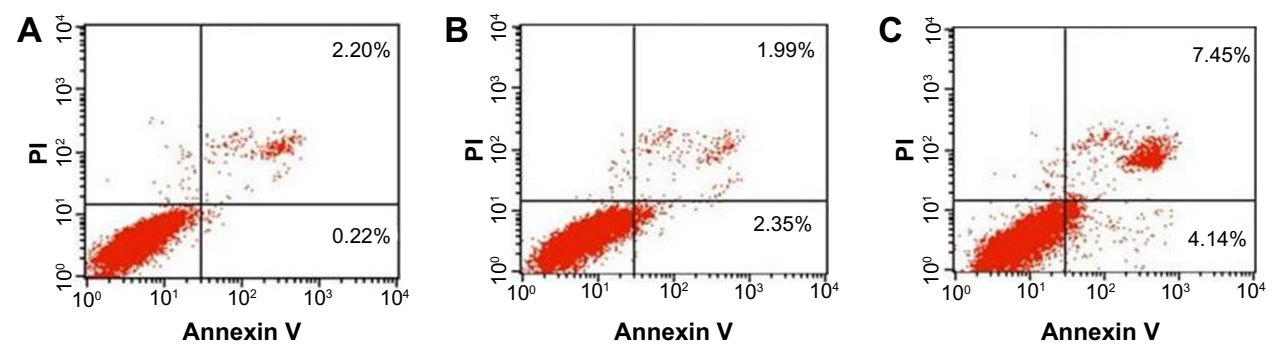

Figure 5 Flow cytometric analysis of fluorescein isothiocyanate annexin $\vee$ staining. Human MDA-MB-23I breast cancer cells were left untreated (A), or treated for 72 hours with nanohydroxyapatites (B), or nanobacteria (C).

Abbreviation: PI, propidium iodide.

into intracellular endosomes. The changes that were characteristic for apoptosis, such as cell shrinkage, chromatin condensation, nuclear fragmentation, nuclear dissolution, and the formation of apoptotic bodies, were visible after 72 hours of NB treatment but were absent in the nHAP and control groups. In addition, mitochondrial swelling was observed in both treatment groups, and it was particularly pronounced in the NB group (Figure 6).

\section{Discussion}

Although some researchers have expressed doubts regarding whether NB are alive or not, ${ }^{19,20}$ our results suggest that they are self-propagating microorganisms: NB divided when the cells were cultured, and many NB particles in culture were similar to cells undergoing binary fission, as observed by TEM (Figure 7). Previous studies demonstrated that nanoparticles inhibit the proliferative activity of a number of mammalian cells. ${ }^{21,22}$ The data presented here clearly show that both NB and nHAPs induced an inhibitory effect on the proliferation of MDA-MB-231 human breast cancer cells. From the results presented here, it is evident that NB exerted more potent cytotoxic effects on MDA-MB-231 cells than nHAPs, and that this may in part be explained by the toxicity of biomembranes, as well as by that of NB metabolite ${ }^{23}$ such as endotoxins, lipopolysaccharides, interleukins, heat shock proteins, matrix metalloproteinases, and so on. ${ }^{1}$

To investigate whether the nanoparticle-induced cytotoxicity in MDA-MB-231 cells is mediated by apoptosis
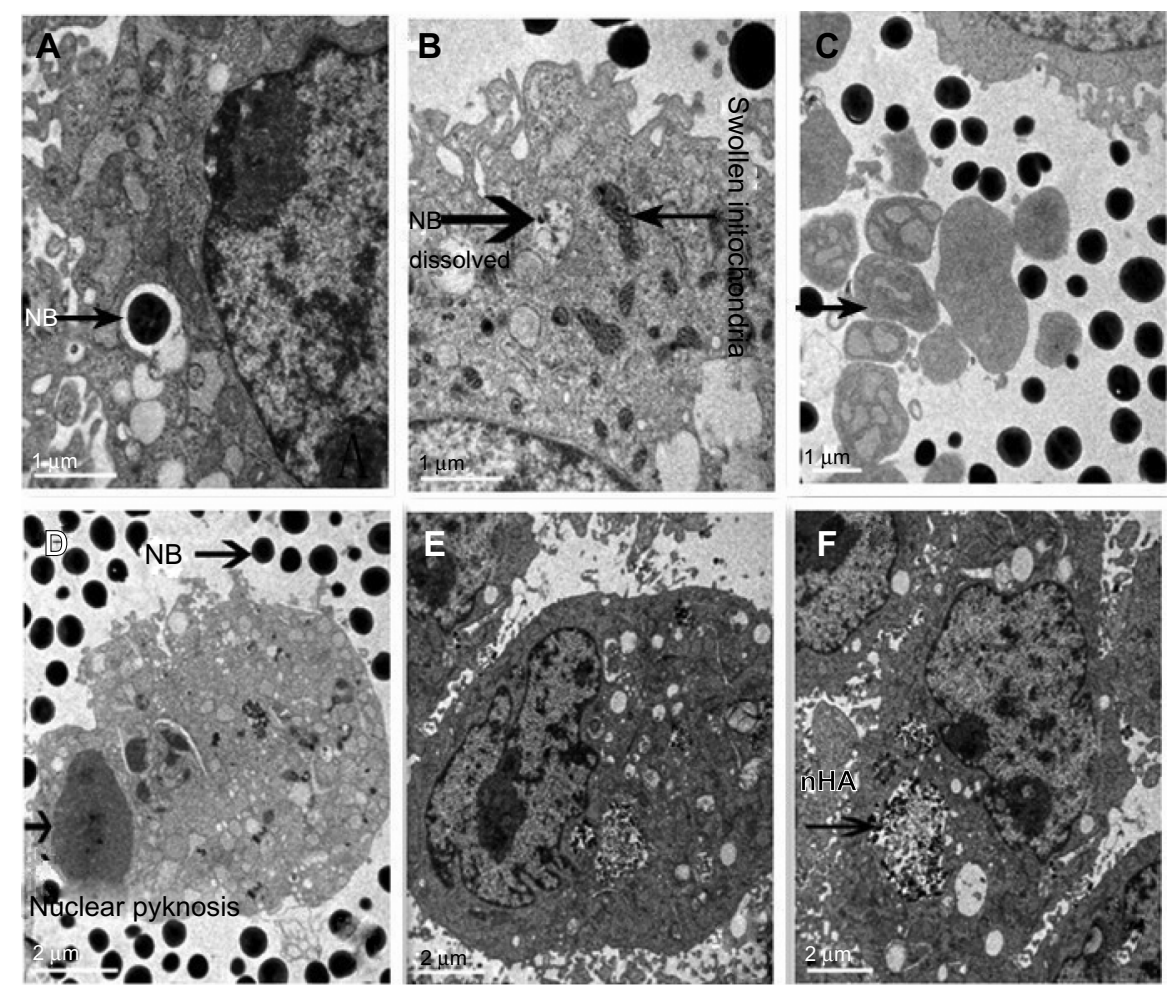

Figure 6 Transmission electron microscopy showing the uptake of nanobacteria (NB) by DA-MB-23I cells.

Notes: (A) NB; (B) NB dissolved and swollen mitochondria; (C) arrow indicates apoptotic bodies; (D) karyopyknosis; (E and F) cells attacked by nanohydroxyapatites (nHA). 


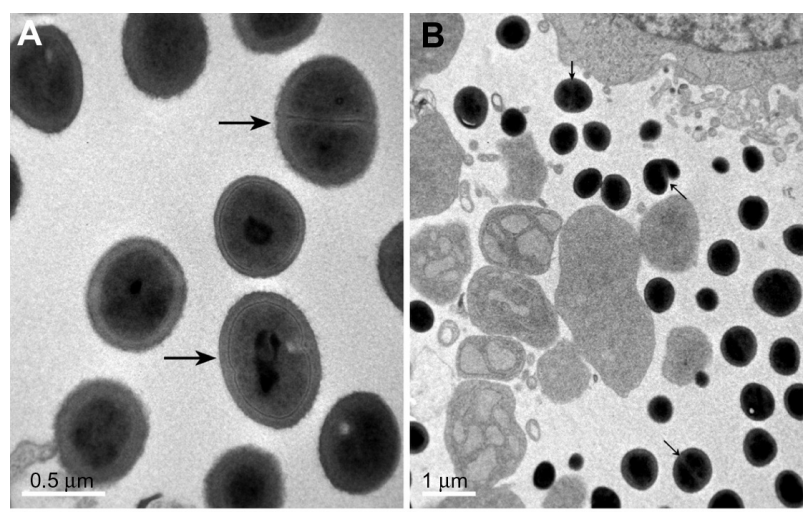

Figure 7 Morphology (binary fission) of nanobacteria under transmission electron microscopy.

Notes: (A) arrows mean binary fission of pure nanobacteria. (B) arrows mean binary fission of nanobacteria attacked MDA-MB-23। cells.

and/or necrosis, we performed the annexin V-FITC/PI assay by flow cytometry and examined the ultrastructure of MDA-MB-231 cells by TEM. The annexin V-FITC/PI assay revealed an increase in early apoptotic cell populations after NB or nHAP exposure. An increase in late apoptotic or dead cell populations (annexin V-positive and PI-positive cells) was also found in the NB but not in the nHAPs group, probably because of the mechanisms we discussed earlier. More important, TEM revealed characteristic apoptotic changes such as mitochondrial swelling, karyopyknosis, karyorrhexis, and karyolysis, along with the breakdown of cells into multiple spherical bodies (apoptotic bodies). Thus, we believe that the toxicity of NB to MDA-MB-231 human breast cancer cells is associated with apoptosis.

To the best of our knowledge, no published data were available regarding the apoptotic potential of NB to human breast cancer cell lines. Here, we report for the first time the cytotoxic effects of NB on MDA-MB-231 cells via the induction of apoptosis. We assume that NB trigger apoptosis in MDA-MB-231 cells by activating a specific signaling pathway after being internalized into a subcellular compartment. In mammalian cells, apoptosis is regulated by the activation of two signaling pathways: an extrinsic one and an intrinsic one. The extrinsic pathway is regulated by death receptors on the plasma membrane. In this pathway, ligand binding to the receptors induces the activation of the caspase cascade. The intrinsic pathway is regulated by mitochondrial proteins. ${ }^{24-26}$ According to the theory of matrix vesicles of apoptotic bodies, apoptosis may alter calcium homeostasis and promote tissue calcification. ${ }^{27}$ Further studies are required to elucidate the precise mechanisms by which nanoparticles initiate and regulate apoptotic cell death.

The following measures have been taken in this study to prevent the contamination of cell cultures and to generate consistent, reliable results: we ensured that all cell culture materials were sterile and that all operations were performed in the hood, using strict aseptic techniques; NB and nHAP solutions and cell culture media were incubated at $56^{\circ} \mathrm{C}$ for 30 minutes to inactivate mycoplasma and Chlamydia; all cell culture solutions were sterilized by filtration through a $0.22 \mu \mathrm{m}$ membrane filter; and the study included welldesigned control groups that were treated exactly in the same way as the experimental groups, except for the variables tested (NB and nHAPs).

\section{Conclusion}

In this study, we described for the first time the in vitro effects of NB and nHAPs on human breast cancer cells. Although both were shown to induce cytotoxicity in MDAMB-231 cells through apoptosis, the effects exerted by NB were more potent. NB cytotoxicity probably arises from its toxic metabolites or protein components, rather than only the hydroxyapatite shells. NB divided during culturing, and many NB particles were observed in culture, similar to cells undergoing binary fission, by TEM. This suggested that NB are live microorganisms.

\section{Acknowledgment}

This study was supported by the National Natural Science Foundation for Young Scholars of China (31000075). We express our gratitude to the Institute of Life Sciences of Chongqing Medical University for help in observing the morphology of MDA-MB-231 cells under TEM and for detecting the apoptosis rate of MDA-MB-231 cells.

\section{Disclosure}

The authors report no conflicts of interest in this work.

\section{References}

1. Kajander EO. Nanobacteria - propagating calcifying nanoparticles. Lett Appl Microbiol. 2006;42(6):549-552.

2. Kutikhin AG, Brusina EB, Yuzhalin AE. The role of calcifying nanoparticles in biology and medicine. Int J Nanomedicine. 2012;7:339-350.

3. Martel J, Young JD. Purported nanobacteria in human blood as calcium carbonate nanoparticles. Proc Natl Acad Sci U S A. 2008;105(14): $5549-5554$.

4. Martel J, Wu CY, Young JD. Critical evaluation of gamma-irradiated serum used as feeder in the culture and demonstration of putative nanobacteria and calcifying nanoparticles. PLoS One. 2010;5(4): e10343.

5. Lu H, Guo YN, Liu SN, Zhang DC. Nanobacteria may be linked to calcification in placenta. Ultrastruct Pathol. 2012;36(3):160-165.

6. Mathew G, Mckay DS, Ciftçioglu N. Do blood-borne calcifying nanoparticles self-propagate? Int J Nanomedicine. 2008(2):265-275.

7. Cisar JO, Xu DQ, Thompson J, Swaim W, Hu L, Kopecko DJ. An alternative interpretation of nanobacteria-induced biomineralization. Proc Natl Acad Sci U S A. 2000;97(21):11511-11515. 
8. Hu YR, Zhao Y, Sun YW, et al. Detection of nanobacteria-like material from calcified cardiac valves with rheumatic heart disease. Cardiovasc Pathol. 2010;19(5):286-292.

9. Schlieper G, Krüger T, Heiss A, Jahnen-Dechent W. A red herring in vascular calcification: 'nanobacteria' are protein-mineral complexes involved in biomineralization. Nephrol Dial Transplant. 2011;26(11): 3436-3439.

10. Raoult D, Drancourt M, Azza S, et al. Nanobacteria are mineralo fetuin complexes. PLoS Pathog. 2008;4(2):e41.

11. Urbano P, Urbano F. Nanobacteria: facts or fancies? PLoS Pathog. 2007;3(5):e55.

12. Young JD, Martel J, Young D, et al. Characterization of granulations of calcium and apatite in serum as pleomorphic mineralo-protein complexes and as precursors of putative nanobacteria. PLoS One. 2009;4(5): e5421.

13. Wang L, Shen W, Wen J, An X, Cao L, Wang B. An animal model of black pigment gallstones caused by nanobacteria. Dig Dis Sci. 2006;51(6):1126-1132.

14. Khullar M, Sharma SK, Singh SK, et al. Morphological and immunological characteristics of nanobacteria from human renal stones of a north Indian population. Urol Res. 2004;32(3):190-195.

15. Jelic TM, Roque R, Yasar U, et al. Calcifying nanoparticles associated encrusted urinary bladder cystitis. Int J Nanomedicine. 2008;3(3): 385-390

16. Jones JA, Ciftcioglu N, Schmid JF, Barr YR, Griffith D. Calcifying nanoparticles (nanobacteria): an additional potential factor for urolithiasis in space flight crews. Urology. 2009;73(1):210. e11-e13.

17. Yang F, Zeng J, Zhang W, Sun X, Ling J. Evaluation of the interaction between calcifying nanoparticles and human dental pulp cells: a preliminary investigation. Int J Nanomedicine. 2010;6:13-18.
18. Sedivy R, Battistutti WB. Nanobacteria promote crystallization of psammoma bodies in ovarian cancer. APMIS. 2003;111(10): 951-954.

19. Guo Y, Zhang D, Lu H, Luo S, Shen X. Association between calcifying nanoparticles and placental calcification. Int $J$ Nanomedicine. 2012;7:1679-1686.

20. Shiekh FA. Do calcifying nanoparticles really contain $16 \mathrm{~S}$ rDNA? Int J Nanomedicine. 2012;7:5051-5052.

21. Bratos-Pérez MA, Sánchez PL, García de Cruz S, et al; Grupo AORTICA (Grupo de Estudio de la Estenosis Aórtica). Association between self-replicating calcifying nanoparticles and aortic stenosis: a possible link to valve calcification. Eur Heart J. 2008;29(3):371-376.

22. Schwartz MA, Lieske JC, Kumar V, Farell-Baril G, Miller VM. Humanderived nanoparticles and vascular response to injury in rabbit carotid arteries: proof of principle. Int J Nanomedicine. 2008;3(2):243-248.

23. Schwartz MK, Hunter LW, Huebner M, Lieske JC, Miller VM Characterization of biofilm formed by human-derived nanoparticles. Nanomedicine (Lond). 2009;4(8):931-941.

24. Fulda S. Targeting apoptosis signaling pathways for anticancer therapy. Front Oncol. 2011;27(1):23

25. Shanahan CM, Crouthamel MH, Kapustin A, Giachelli CM. Arterial calcification in chronic kidney disease: key roles for calcium and phosphate. Circ Res. 2011;109(6):697-711.

26. George A, Veis A. Phosphorylated proteins and control over apatite nucleation, crystal growth, and inhibition. Chem Rev. 2008;108(11): 4670-4693.

27. Ronchetti I, Boraldi F, Annovi G, Cianciulli P, Quaglino D. Fibroblast involvement in soft connective tissue calcification. Front Genet $2013 ; 4: 22$
International Journal of Nanomedicine

\section{Publish your work in this journal}

The International Journal of Nanomedicine is an international, peerreviewed journal focusing on the application of nanotechnology in diagnostics, therapeutics, and drug delivery systems throughout the biomedical field. This journal is indexed on PubMed Central,

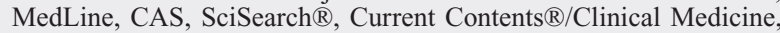

\section{Dovepress}

Journal Citation Reports/Science Edition, EMBase, Scopus and the Elsevier Bibliographic databases. The manuscript management system is completely online and includes a very quick and fair peer-review system, which is all easy to use. Visit http://www.dovepress.com/ testimonials.php to read real quotes from published authors. 